\title{
Burden of illness among patients with dementia-related psychosis
}

\section{What is already known about this subject}

- The presence of neuropsychiatric symptoms in patients with dementia is associated with greater impairments in activities of daily living, faster cognitive decline, increased health care costs, and worse quality of life and contributes to caregiver fatigue, distress, and depression, resulting in earlier institutionalization of patients in long-term care facilities.

- There are no FDA-approved treatments for dementia-related psychosis (DRP), and off-label treatments have been shown to have minimal clinical benefits coupled with a greater potential for adverse events including stroke, acute cardiovascular events, metabolic events, pulmonary effects, irreversible cognitive decline, and death.

- The social, emotional, and financial impacts of dementia and dementiarelated psychosis are known to be high, but the burden of illness between patients with dementia only and DRP has not been compared.

\section{ABSTRACT}

BACKGROUND: More than 5.6 million Americans suffer from dementia, and that number is expected to double by 2060 . This comes at a considerable burden to the health care system with costs estimated at \$157-\$215 billion in 2010. Depending on dementia type and disease progression, approximately $20 \%-70 \%$ of patients experience dementia-related psychosis (DRP), characterized by hallucinations and/or

\section{What this study adds}

- This study documented the higher rates of clinical events, health care resource utilization, and associated health care costs among patients with DRP compared with those with dementia only.

- Patients with DRP had significantly more falls/fractures and sedation compared with patients with dementia only and consistently higher all-cause and dementia-related health care utilization and costs, driven by acute care received in emergency and inpatient settings, compared with patients with dementia only.

- Study findings suggest that patients with DRP experience a greater burden of illness compared with patients with dementia only. delusions resulting in worse clinical outcomes and greater caregiver burden compared with patients without DRP.

OBJECTIVE: To compare real-world clinical events, health care resource utilization (HCRU), and health care costs among matched cohorts of DRP versus dementiaonly patients.

METHODS: This retrospective database analysis examined commercial and Medicare Advantage with Part D enrollees aged $\geq 40$ years with evidence of DRP and dementia

\section{Author affiliations}

Monica Frazer, PhD, and Rachel Halpern, $\mathrm{PhD}$, Optum, Eden Prairie, MN. Victor Abler, DO; Ben Skoog, PharmD; and Nazia Rashid, PharmD, Acadia Pharmaceuticals, San Diego, CA.

AUTHOR CORRESPONDENCE: Monica Frazer, 952.205.8356; monica.frazer@optum.com

J Manag Care Spec Pharm. 2021;27(3):367-78

Copyright $@ 2021$, Academy of Managed Care Pharmacy. All rights reserved.

from January 1, 2010, through March 31, 2017. The first observed indicator of psychosis ( $\geq 2$ diagnoses and/or antipsychotic pharmacy fills) co-occurring with or following evidence of dementia ( $\geq 2$ diagnoses and/ or dementia medication pharmacy fills) was the index date among patients with DRP. DRP patients were propensity score matched 1:1 to patients with dementia only based on demographics, comorbidities, dementia type, dementia severity, and pre-index all-cause HCRU. Continuous health plan enrollment $\geq 12$ months before evidence of dementia 
through the index date and $\geq 12$ months following the index date was required. Outcomes included clinical events, HCRU, and health care costs.

RESULTS: A significantly higher percentage of DRP patients had $\geq 1$ diagnosis for behavioral health conditions in the pre-index period compared with dementia-only patients (depression: $32.4 \%$ vs. $22.8 \%$; anxiety: $19.1 \%$ vs. $11.5 \%$; and insomnia: $9.0 \%$ vs. $6.3 \% ; P<0.001$ for all comparisons). Diagnoses of post-index clinical events were significantly more likely among DRP patients compared with dementia-only patients including falls/fractures ( $28.3 \%$ vs. $14.1 \%)$, neurologic effects ( $17.7 \%$ vs. $12.9 \%)$, sedation ( $15.0 \%$ vs. $2.4 \%$ ), cardiovascular effects (7.0\% vs. $4.1 \%$ ), and extrapyramidal reactions (3.2\% vs. $1.7 \% ; P<0.001$ for all comparisons). Higher percentages of DRP patients had an all-cause outpatient visit ( $80.2 \%$ vs. $68.9 \%)$, emergency visit $(65.0 \%$ vs. $36.6 \%)$, or inpatient stay ( $47.2 \%$ vs. $20.0 \%$ ) during the post-index period ( $\mathrm{P}<0.001$ for all comparisons). The proportions of DRP patients with a post-index dementia-related office visit, outpatient visit, emergency visit, or inpatient stay was $48 \%, 147 \%, 339 \%$, and $286 \%$ higher, respectively, compared with patients with dementia only. Compared with patients with dementia only, patients with DRP had significantly higher mean total post-index all-cause costs $(\$ 21,657$ vs. $\$ 12,026$; $P<0.001)$ and dementia-related costs $(\$ 11,852$ vs. $\$ 3,013 ; P<0.001)$.

CONCLUSIONS: Patients with DRP were more likely to have diagnoses for behavioral health conditions, experience clinical events, and have higher mean all-cause and dementia-related HCRU and costs compared with patients with dementia only. These results reflect the unmet need of patients with DRP and an urgency for new treatment options to reduce substantial clinical and economic burden in this population.

Dementia is a chronic condition characterized by memory disorders, personality changes, and impaired reasoning and is thought to affect more than 5.6 million adults in the United States aged 65 years and older. The prevalence of dementia is likely underestimated ${ }^{1-4}$ with 1 study documenting that $59 \%$ of patients who met diagnostic criteria for dementia were undiagnosed or unaware of their diagnosis. ${ }^{5}$ As the population ages, it has been estimated that the number of new cases of dementia diagnosed among individuals aged older than 65 years will double by $2060 .^{6}$

In 2010, the estimated costs associated with dementia in the United States ranged from $\$ 157$ billion to $\$ 215$ billion, of which Medicare paid approximately $\$ 11$ billion. ${ }^{7}$ In 1 analysis of fee-for-service Medicare claims, $10 \%$ of elderly Medicare beneficiaries had dementia with mean costs 3 times higher than those for beneficiaries without dementia (\$13,207 vs. $\$ 4,454)$. Additionally, costs associated with coronary heart disease, congestive heart failure, diabetes, and chronic obstructive pulmonary disease were 2 times higher in patients with dementia compared with those without. ${ }^{8}$

Dementia is most commonly diagnosed as Alzheimer disease but can also take other forms including vascular dementia, dementia with Lewy bodies, dementia related to Parkinson disease, frontotemporal dementia (i.e., Pick disease), other dementias (i.e., senile, presenile dementias), and mixed dementia (a combination of 2 or more dementia types). Depending on dementia type and increasing with disease progression, approximately $20 \%-70 \%$ of patients will experience dementia-related psychosis (DRP), characterized by delusions and hallucinations. ${ }^{9}$ This estimate is thought to increase to more than $90 \%$ if behavioral symptoms such as dementia-related agitation and aggression are included as indicators of psychosis..$^{10}$ In a study by Peters et al. (2015), DRP was associated with a more rapid progression to severe dementia (hazard ratio $=2.007 ; P=0.028$ ) and earlier death (hazard ratio $=1.537 ; \mathrm{P}=0.011$ ) among patients with Alzheimer disease. ${ }^{11}$ Neuropsychiatric symptoms not only present a considerable burden to patients including greater impairments in activities of daily living, ${ }^{12}$ more rapid cognitive decline, ${ }^{12,13}$ and worse quality of life, ${ }^{14}$ but they also contribute to caregiver fatigue and depression, ${ }^{15}$ resulting in earlier institutionalization of the patient in long-term care facilities. ${ }^{13,16}$ In a study by Maust et al. (2017), caregiver distress related to psychosis or agitation in a patient with dementia was associated with increased utilization of emergency services, inpatient hospitalization, and Medicare expenditures. ${ }^{17}$

Currently, there are no treatments for DRP that are approved by the U.S. Food and Drug Administration, so patients with DRP are often treated with off-label antipsychotic medication when symptoms become severe, dangerous, or cause significant distress to the patient. ${ }^{18}$ Several clinical trials have noted minimal clinical benefits of antipsychotics on symptoms of DRP; however, the potential for adverse events, including stroke, acute cardiovascular events, metabolic events, pulmonary effects, irreversible cognitive decompensation, and death, is great. ${ }^{19-24}$

Due to the increasing prevalence of dementia and the unmet need of an effective safe treatment option for DRP, it is important for payers and providers to understand the real-world clinical and economic burden of patients with DRP. The purpose of this study was to compare the clinical events, health care resource utilization (HCRU), and health care costs between dementia patients with DRP and patients with dementia only. 


\section{Methods}

\section{DATA SOURCE}

This was a retrospective study using medical and pharmacy claims and enrollment information from commercial or Medicare Advantage with Part D enrollees in the Optum Research database from January 1, 2008, to March 31, 2018 (study period). The Optum Research database is geographically diverse, representing approximately $19 \%$ of the commercially enrolled population and $21 \%$ of the Medicare Advantage population in the United States as of 2018. To classify inpatient and outpatient services, International Classification of Diseases, Ninth/Tenth Revisions, Clinical Modification diagnosis codes and procedure codes, Current Procedural Terminology 4th Edition procedure codes, Healthcare Common Procedure Coding system codes, and place-of-service codes were used, all of which are found on medical claims. Outpatient pharmacy claims were classified using National Drug Codes for dispensed medications, quantity dispensed, dose, and days' supply.

\section{STUDY SAMPLE SELECTION}

Patients were assigned to cohorts based on the presence (DRP cohort) or absence (dementia-only cohort) of claims indicating psychosis (Supplementary Table 1, available in online article). For inclusion in the DRP cohort, patients met at least 1 of the 3 following criteria: (1) $\geq 2$ medical claims with a psychosis diagnosis in any position on the claim $\geq 30$ days and $\leq 365$ days apart; (2) $\geq 2$ pharmacy fills $\leq 60$ days apart or $\geq 1$ pharmacy fill with $\geq 45$ days' supply for a DRP therapy (atypical or typical first-generation antipsychotic, divalproex sodium, or dextromethorphan/quinidine); or (3) $\geq 1$ medical claim with a psychosis diagnosis code in any position and $\geq 1$ pharmacy claim for a DRP therapy $\leq 365$ days apart. The date of the first psychosis diagnosis or DRP therapy fill between January 1, 2010, and March 31, 2017, (identification period) was the index date.

Additionally, DRP patients must have had evidence of dementia in the 12 months before or on the index date, meeting at least 1 of the following 3 criteria: (1) $\geq 2$ medical claims $\geq 30$ days apart with a diagnosis of Alzheimer disease, vascular dementia, dementia with Lewy bodies, dementia with Parkinson disease, frontotemporal dementia (i.e., Pick disease and other frontotemporal dementias), other dementia (i.e., presenile, senile, or dementia in other diseases that were not Parkinson disease), or unspecified dementia diagnoses in any position on the claim; (2) $\geq 2$ pharmacy fills for dementia therapy (i.e., donepezil, galantamine, memantine, rivistigmine, tacrine) $\leq 60$ days apart or $\geq 1$ pharmacy fill with $\geq 45$ days' supply; or (3) $\geq 1$ medical claim with a dementia diagnosis and $\geq 1$ pharmacy claim for dementia-related therapy $\leq 365$ days apart (Supplementary Table 1, available in online article). The first dementia diagnosis or dementia-related pharmacy fill during the preindex period was the baseline dementia date. Continuous enrollment with medical and pharmacy benefits for $\geq 12$ months before the baseline dementia date (predementia period) until the index date and $\geq 12$ months including and following the index date (post-index period) was required. Patients included in the analyses were aged $\geq 40$ years during the index year.

DRP patients were excluded from the study if they had a medical claim with a diagnosis code in any position during the pre-index period for any of the following conditions: a mental health condition manifesting as psychosis; substance use, abuse, or dependence associated with psychosis; psychosis due to delirium; cerebral amyloid angiopathy; central nervous system neoplasm, intracranial mass lesion, or vascular malformation; ischemic or hemorrhagic stroke; and cortical degeneration.

DRP patients were also excluded if they had a medical claim with a diagnosis code for pseudobulbar affect in any position on the claim and were treated with dextromethorphan or a medical claim with a diagnosis code for epilepsy or seizure in any position on the claim and were treated with divalproex during the pre-index or post-index periods. Patients in the DRP cohort were excluded if they had a medical claim for agitation/aggression during the 12-month pre-index period, if they had a medical claim with a psychosis diagnosis or $\geq 2$ pharmacy fills for a DRP-related therapy $\geq 12$ months before the baseline dementia date through the index date, or if they had a pharmacy claim for pimavanserin during the study period.

To be included in the dementia-only cohort, patients must have had evidence of dementia as described above during the identification period. The $\geq 2$ dementia diagnoses or $\geq 1$ dementia diagnosis and $\geq 1$ dementia-related medication fill must have occurred within 24 months of each other; the date of the earliest claim was the dementia date. Patients in the dementia-only cohort were required to have $\geq 12$ months of continuous enrollment with medical and pharmacy benefits before and following the dementia date and be aged $\geq 40$ years as of the dementia date year. Dementia-only patients must have had no evidence of psychosis or agitation/aggression during the pre-index or post-index periods. Exclusion and continuous enrollment criteria as described above were applied.

\section{PROPENSITY SCORE MATCHING}

Patients in the DRP cohort were propensity score matched to patients in the dementia-only cohort based on demographic characteristics (age, sex, insurance type, region); 
comorbidities; type of dementia; pre-index all-cause HCRU; and dementia severity indicated by therapy prescription (mild/moderate vs. moderate/severe based on the therapy indications in the prescribing instructions) in the 12 months before the dementia date. Dementia-only patients with the closest available propensity score within a caliper of \pm 0.01 were matched N:1 to patients in the DRP cohort. Index dates for dementia-only patients were assigned so that the time between the baseline dementia date and the index date equaled that of their matched DRP patient. Once dementiaonly patients were assigned an index date, a requirement for 12 months of post-index continuous enrollment was applied. The 1:1 match was conducted by choosing dementia-only patients whose propensity score was closest to their DRP match. If there was more than 1 potential match, the dementia-only patient with the closest index year was chosen as the 1:1 match. Following propensity score matching, bivariate comparisons of demographics and pre-index characteristics were conducted to assess the success of the matching procedure. Standardized differences between cohorts for all measures were below $6.0 \%$ postmatch.

\section{STUDY MEASURES}

Demographic and Clinical Characteristics. Demographic and clinical characteristics including age; gender; insurance type; geographic region; Charlson Comorbidity Index ${ }^{25,26}$; select comorbidities (anxiety, depression, and insomnia disorders); and dementia type were measured during the patients' 12-month pre-index period.

Outcomes. For this study, 3 outcomes were measured: clinical events, health care resource utilization (HCRU), and health care costs.

1. Clinical events were measured in the 12-month postindex period and were defined as the proportion of patients with $\geq 1$ medical claim with a diagnosis in any position on the claim for extrapyramidal reactions (e.g., tremor, tics, dystonia); tardive dyskinesia; neurologic effects (e.g., headache, dizziness, convulsions); anticholinergic effects (e.g., dry mouth, visual disturbance, constipation); cardiovascular effects (e.g., myocardial or cerebral infarction, embolism, orthostatic hypertension); sedation; falls/fractures; or medication-related cognitive effects $(\geq 1$ diagnosis code for cognitive decline and $\geq 1$ diagnosis code indicating an adverse effect due to medication use on the same claim). Clinical events were not measured with any temporal relationship to antipsychotic consumption.

2. All-cause HCRU was measured as the number of visits and the proportion of patients with $\geq 1$ office visit, outpatient visit, emergency visit, or inpatient stay during the 12-month pre-index and post-index periods. Dementiarelated HCRU, measured in the post-index period, was defined as above and considered dementia related if the medical claim had a diagnosis code for dementia in any position or was a pharmacy fill for a dementia medication. 3. All-cause health care costs were calculated as the combined health plan and patient-paid amounts in the 12-month pre-index and post-index periods adjusted for inflation from 2008 to 2017 using the annual medical care component of the Consumer Price Index..$^{27}$ Cost categories included office, outpatient, emergency, inpatient, other medical costs (i.e., durable medical equipment), and pharmacy costs. Dementia-related costs were calculated as above in the post-index period and were considered dementia related if the claim had a diagnosis code for dementia in any position or if the pharmacy claim was for a dementia-related medication.

\section{ANALYSIS}

Descriptive analyses were conducted for all pre-index and post-index measures. Outcome comparisons were conducted based on the distribution of the measure. In comparisons between matched cohorts, Rao-Scott test for binary measures and Z-test using robust standard errors in an ordinary least squares regression for continuous measures were used. In comparisons between pre-index and post-index HCRU and costs within the DRP cohort, McNemar's test for binary and 2-sample t-test for continuous measures were used.

\section{Results}

\section{PRE-INDEX DEMOGRAPHICS AND CLINICAL CHARACTERISTICS}

There were 12,021 patients with DRP matched to 12,021 patients with dementia only who were included in the study analysis (Figure 1). After match, the distribution of patients across age, gender, and insurance type was similar between the cohorts: mean age differed by one half of a year (80.1 years DRP, 79.6 years dementia only), two thirds were female (66.4\% DRP; $66.2 \%$ dementia only), and most were enrolled in Medicare Advantage with Part D (86.3\% DRP; 85.7\% dementia only; Table 1). Patients with DRP were more likely to have $\geq 1$ diagnosis for a depressive disorder $(32.4 \%$ vs. $22.8 \% ; \mathrm{P}<0.001)$, anxiety disorder $(19.1 \%$ vs. $11.5 \%$; $P<0.001)$, and insomnia disorder $(9.0 \%$ vs. $6.3 \%$; $\mathrm{P}<0.001)$ compared with dementia-only patients. Patients with dementia diagnoses could have received more than 1 type of diagnosis; the most common dementia diagnoses were unspecified or other dementia (84.0\% DRP; $62.3 \%$ 


\section{FIGURE 1 Patient Sample Selection}

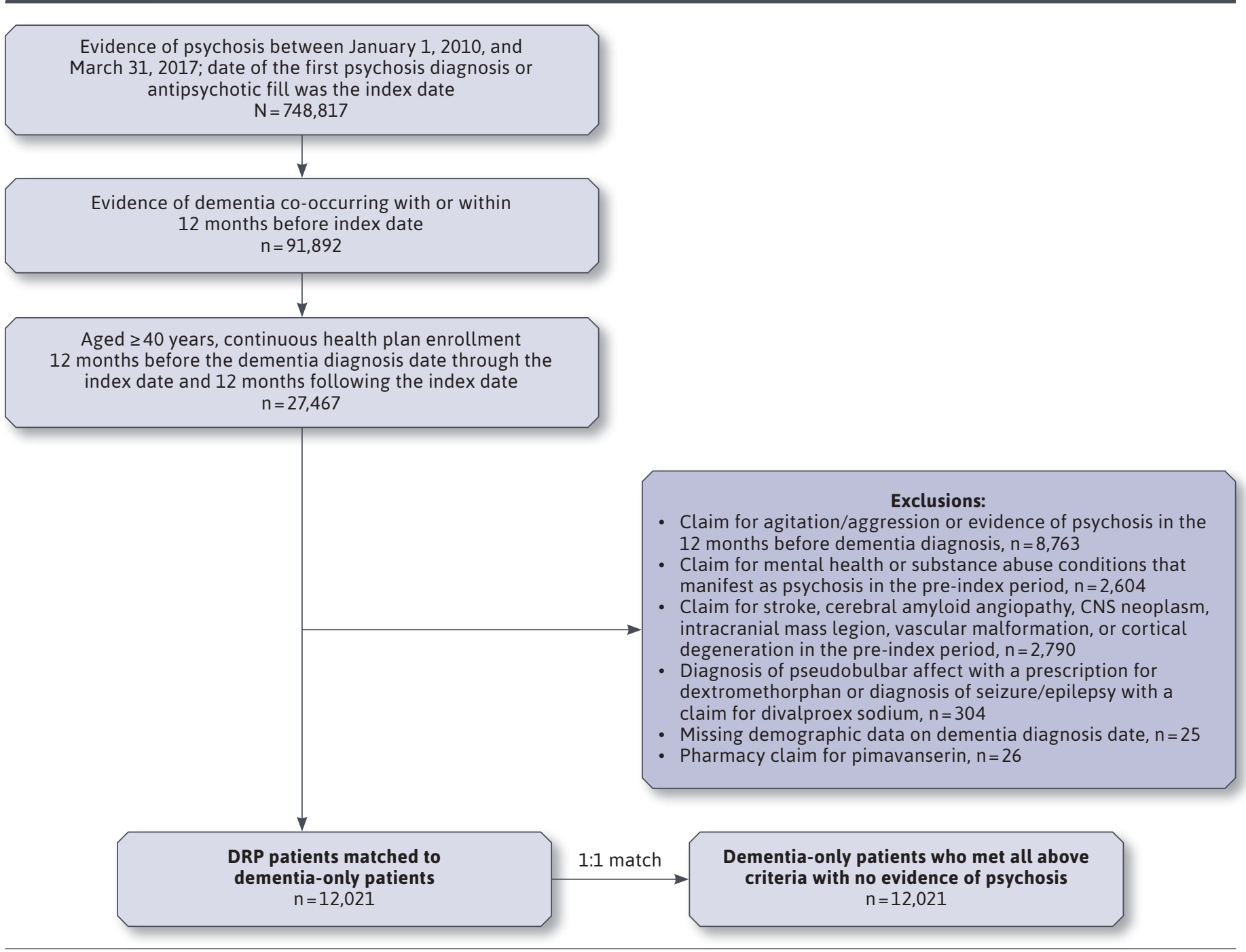

CNS = central nervous system; $D R P=$ dementia-related psychosis .

dementia only; $\mathrm{P}<0.001)$ and Alzheimer disease $(40.7 \%$ DRP; 29.4\% dementia only; $\mathrm{P}<0.001)$.

\section{CLINICAL EVENTS}

Falls/fractures were the most common clinical event for all patients, with $28.3 \%$ of DRP patients and $14.1 \%$ of dementia-only patients having received $\geq 1$ diagnosis for a fall or fracture during the post-index period (Figure 2). In addition, patients in the DRP cohort also had higher rates of neurologic effects $(17.7 \%$ vs. $12.9 \%$; $\mathrm{P}<0.001)$, sedation (15.0\% vs. 2.4\%; P<0.001), cardiovascular effects $(7.0 \%$ vs.
4.1\%; $\mathrm{P}<0.001)$, and extrapyramidal reactions $(3.2 \%$ vs. $1.7 \% ; \mathrm{P}<0.001)$.

\section{HEALTH CARE RESOURCE UTILIZATION}

In the pre-index period, a significantly higher percentage of DRP than dementia-only patients had an all-cause emergency visit $(49.1 \%$ vs. $36.7 \%$; $P<0.001)$ or inpatient stay (27.4\% vs. $18.9 \%$; $\mathrm{P}<0.001)$, whereas a higher percentage of dementia-only patients had an all-cause office visit (91.8\% vs. 89.9\%; P<0.001; Figure 3). Patients with DRP had a higher mean (SD) number of pre-index all-cause emergency 


\section{TABLE 1 Pre-Index Demographic and Clinical Characteristics}

\begin{tabular}{|c|c|c|c|c|c|c|c|}
\hline \multirow[b]{2}{*}{ Age, mean (SD), years } & \multicolumn{2}{|c|}{$\begin{array}{c}\text { Total } \\
(\mathrm{N}=\mathbf{2 4 , 0 4 2})\end{array}$} & \multicolumn{2}{|c|}{$\begin{array}{c}\text { DRP } \\
(n=12,021)\end{array}$} & \multicolumn{2}{|c|}{$\begin{array}{l}\text { Dementia Only } \\
\qquad(n=12,021)\end{array}$} & \multirow{2}{*}{$\begin{array}{l}\text { P Value } \\
<0.001\end{array}$} \\
\hline & 79.9 & $(7.2)$ & 80.1 & $(6.9)$ & 79.6 & (7.4) & \\
\hline Female gender, n (\%) & 15,939 & $(66.3)$ & 7,981 & $(66.4)$ & 7,958 & $(66.2)$ & 0.751 \\
\hline \multicolumn{8}{|l|}{ Insurance type, n (\%) } \\
\hline Commercial & 3,364 & $(14.0)$ & 1,646 & $(13.7)$ & 1,718 & $(14.3)$ & 0.174 \\
\hline Medicare Advantage with Part D & 20,678 & $(86.0)$ & 10,375 & $(86.3)$ & 10,303 & $(85.7)$ & 0.174 \\
\hline \multicolumn{8}{|l|}{ Region, n (\%) } \\
\hline Northeast & 4,258 & $(17.7)$ & 2,192 & $(18.2)$ & 2,066 & $(17.2)$ & 0.031 \\
\hline Midwest & 7,712 & $(32.1)$ & 3,763 & $(31.3)$ & 3,949 & $(32.9)$ & 0.008 \\
\hline South & 9,368 & $(39.0)$ & 4,753 & $(39.5)$ & 4,615 & $(38.4)$ & 0.063 \\
\hline West & 2,701 & $(11.2)$ & 1,312 & $(10.9)$ & 1,389 & $(11.6)$ & 0.112 \\
\hline Othera $^{\mathrm{a}}$ & 3 & $(0.01)$ & 1 & $(0.01)$ & 2 & $(0.02)$ & 0.564 \\
\hline Charlson Comorbidity Index, mean (SD) & 1.8 & $(1.8)$ & 1.9 & $(1.9)$ & 1.7 & $(1.8)$ & $<0.001$ \\
\hline \multicolumn{8}{|l|}{ Comorbid disorders ${ }^{\mathrm{b}}, \mathrm{n}(\%)$} \\
\hline Depressive disorder & 6,643 & $(27.6)$ & 3,898 & $(32.4)$ & 2,745 & $(22.8)$ & $<0.001$ \\
\hline Anxiety disorder & 3,684 & $(15.3)$ & 2,298 & $(19.1)$ & 1,386 & $(11.5)$ & $<0.001$ \\
\hline Insomnia disorder & 1,833 & $(7.6)$ & 1,076 & $(9.0)$ & 757 & $(6.3)$ & $<0.001$ \\
\hline \multicolumn{8}{|l|}{ Dementia type ${ }^{\mathrm{b}}, \mathrm{n}(\%)$} \\
\hline Alzheimer disease & 8,423 & $(35.0)$ & 4,894 & $(40.7)$ & 3,529 & $(29.4)$ & $<0.001$ \\
\hline Vascular dementia & 1,824 & $(7.6)$ & 1,062 & $(8.8)$ & 762 & $(6.3)$ & $<0.001$ \\
\hline Dementia with Lewy bodies & 452 & $(1.9)$ & 300 & $(2.5)$ & 152 & $(1.3)$ & $<0.001$ \\
\hline Parkinson disease with dementia & 527 & $(2.2)$ & 366 & (3.0) & 161 & $(1.3)$ & $<0.001$ \\
\hline Frontotemporal dementia & 208 & $(0.9)$ & 131 & $(1.1)$ & 77 & $(0.6)$ & $<0.001$ \\
\hline Unspecified/other dementia & 17,593 & $(73.2)$ & 10,099 & $(84.0)$ & 7,494 & $(62.3)$ & $<0.001$ \\
\hline
\end{tabular}

a"Other" refers to Armed Forces Americas (except Canada), Armed Forces (Europe, Canada, Middle East, Africa), Armed Forces Pacific, American Samoa, Federated State of Micronesia, Guam, Marshall Islands, Commonwealth of the Northern Mariana Islands, Puerto Rico, Palau, Virgin Islands.

${ }^{b} \geq 1$ diagnosis code in any position.

$D R P=$ dementia-related psychosis.

visits $(1.1[2.3]$ vs. 0.7 [2.3]; $\mathrm{P}<0.001)$ and inpatient stays $(0.4$ [0.8] vs. 0.3 [0.6]; $\mathrm{P}<0.001)$ than dementia-only patients. Dementia-only patients, however, had a significantly higher mean (SD) number of all-cause office visits (12.1 [12.4] vs. 10.4 [11.2]; $\mathrm{P}<0.001$; data not shown). Despite matching based on dementia-related HCRU, patients with DRP still had significant, but small, differences in all-cause HCRU.

Post-index all-cause HCRU was higher among DRP patients compared with dementia-only patients across all resource categories, with the exception of office visits (Figure 3). The difference in all-cause HCRU among cohorts was driven by an increase in the percentage of patients with inpatient stays (72.5\% increase) and emergency visits (32.3\% increase) from pre-index to post-index among patients with
DRP. DRP patients had a significantly greater mean (SD) number of outpatient visits (7.4 [10.0] vs. 5.6 [8.7]; $P<0.001)$, emergency visits $(1.9$ [3.6] vs. $0.8[2.2] ; P<0.001)$, and inpatient stays $(0.8[1.1]$ vs. $0.3[0.7] ; P<0.001)$ compared with dementia-only patients. Dementia-only patients, however, had a significantly greater mean number of office visits (11.0 [11.8] vs. $9.7[11.6] ; P<0.001$; data not shown).

Post-index dementia-related HCRU was significantly higher for DRP patients compared with dementia-only patients (Figure 3). Higher percentages of DRP patients had $\geq 1$ dementia-related office visit $(53.8 \%$ vs. $36.4 \%$; $P<0.001)$, outpatient visit $(43.6 \%$ vs. $17.6 \%$; $P<0.001)$, emergency visit $(36.4 \%$ vs. $8.3 \%$; $P<0.001)$, and inpatient stay $(40.6 \%$ vs. $10.5 \%$; $P<0.001)$. The mean (SD) number of post-index 


\section{FIGURE 2 Clinical Events During the 12-Month Post-Index Period}

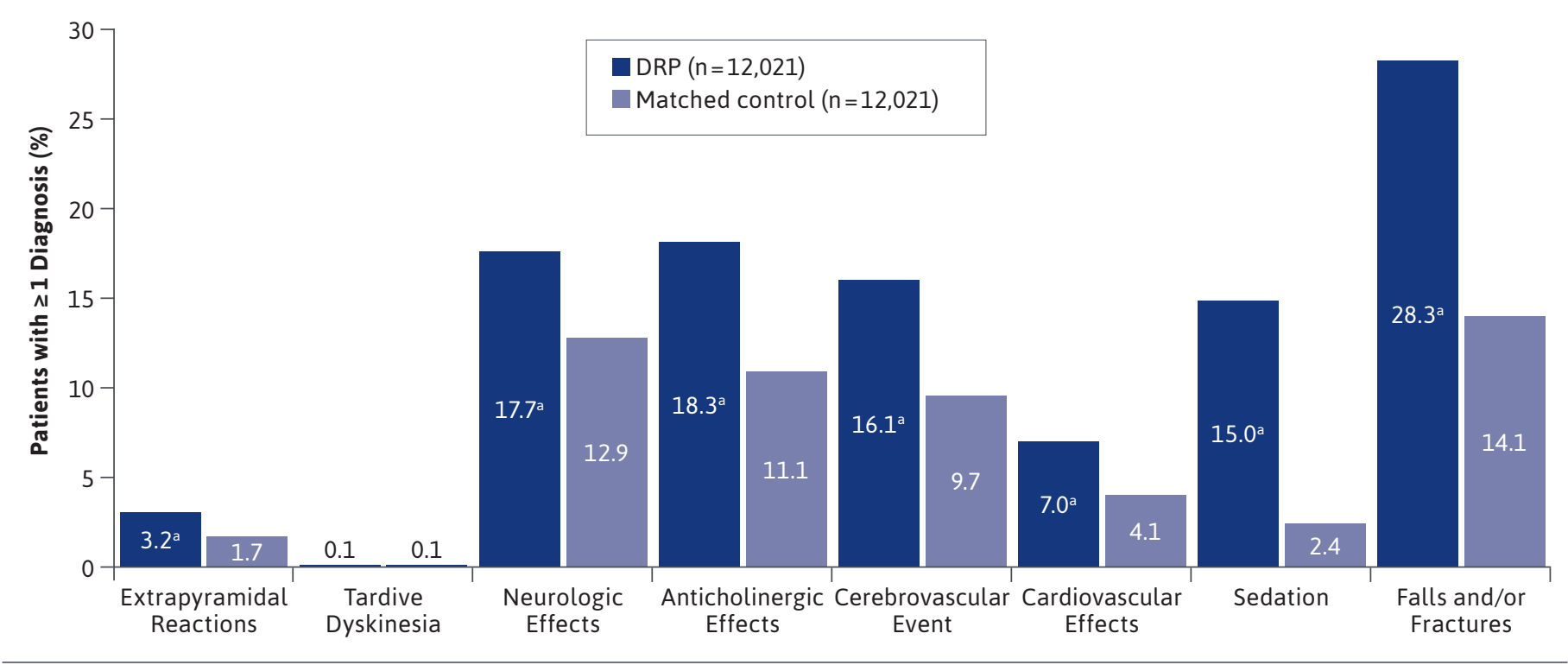

${ }^{a} P<0.001$ for comparison between DRP and dementia only.

$D R P=$ dementia-related psychosis.

dementia-related office visits (1.9 [4.0] vs. 0.8 [1.9]; $P<0.001)$, outpatient visits $(1.9$ [4.9] vs. 0.5 [1.9]; $P<0.001)$, emergency visits $(0.7[1.6]$ vs. $0.1[0.4] ; P<0.001)$, and inpatient stays $(0.6$ $[0.9]$ vs. $0.1[0.4] ; P<0.001)$ were also significantly higher among DRP vs. dementia-only patients.

\section{HEALTH CARE COSTS}

Health care costs for patients in the DRP cohort increased significantly, whereas costs among dementia-only patients remained relatively stable. Patients with DRP had 54.4\% higher health care costs in the post-index period compared with the pre-index period ( $\$ 21,657$ vs. $\$ 14,027 ; \mathrm{P}<0.001)$ versus a $2.6 \%$ difference between post-index and pre-index costs among patients with dementia only (\$12,026 vs. \$11,719; $P=0.096$; Figure 4). The large increase in post-index costs among patients with DRP can be attributed to the $84.3 \%$ increase in inpatient costs from pre-index to post-index ( $\$ 5,704$ vs. $\$ 10,514 ; P<0.001)$, which represented $48.6 \%$ of the post-index cost total. Emergency costs represented a small portion of the cost total $(7.0 \%)$ but increased the greatest percentage from pre-index to post-index (92.3\%) from $\$ 790$ to $\$ 1,519$.

In comparisons across cohorts, mean post-index allcause total health care costs were $80.1 \%$ higher among patients with DRP compared with patients with dementia only $(\mathrm{P}<0.001)$. Compared with dementia-only patients, those with DRP had consistently higher post-index pharmacy costs (29.3\% higher), outpatient costs (25.7\% higher), emergency costs (187.4\% higher), inpatient costs $(180.7 \%$ higher), and other medical costs $(77.8 \%$ higher; $\mathrm{P}<0.001$ for all comparisons).

Total post-index dementia-related costs were significantly higher among DRP versus dementia-only patients (\$11,852 vs. \$3,013; P<0.001; Figure 4). Patients with DRP had higher dementia-related costs across all cost categories. Inpatient costs were the major cost driver, accounting for $73.8 \%$ of dementia-related costs in the DRP cohort and $58.2 \%$ of dementia-related costs among patients in the dementia-only cohort.

\section{Discussion}

Due to the increased life expectancy of older adults, an estimated 13.9 million patients aged 65 years and older will be diagnosed with dementia in the United States by $2060 .^{6}$ With the doubling of the dementia cases, the number of patients experiencing hallucinations and delusions will also increase, emphasizing the importance of understanding the burden of DRP on the health care system. There is a lack of previously published literature investigating the clinical and 


\section{FIGURE 3 Health Care Resource Utilization During the 12-Month Pre-Index and Post-Index Periods ${ }^{\mathrm{a}}$}

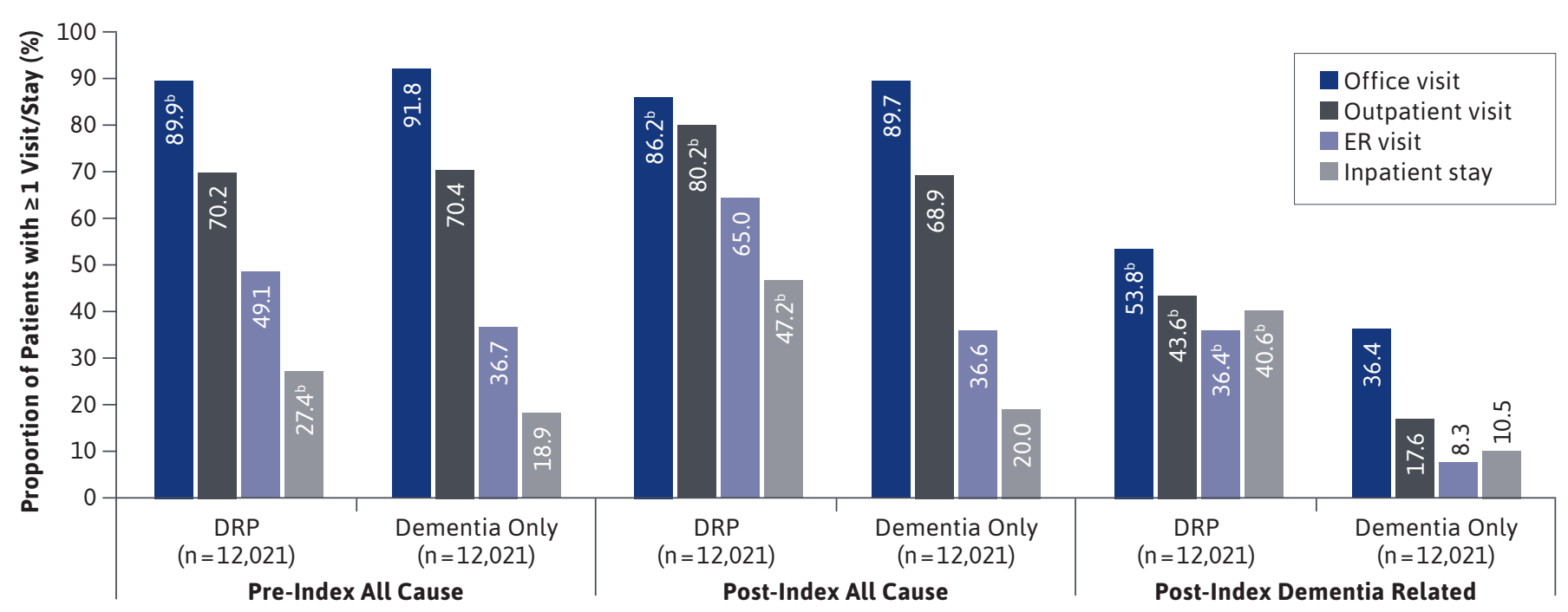

${ }^{a}$ Dementia-related health care utilization was only calculated during the post-index period.

${ }^{b} P<0.001$ for comparison between DRP and dementia only.

$D R P=$ dementia-related psychosis.

economic burden of DRP. This study adds to the current literature gap to address DRP, especially for payers.

A greater proportion of DRP patients experienced a clinical event in the post-index period compared with dementia-only patients. The proportion of DRP patients diagnosed with sedation was more than 6 times higher than the proportion among dementia patients with no psychosis; falls/fractures more than 2 times higher; extrapyramidal reactions almost 2 times higher; and cardiovascular effects 1.7 times higher. Clinical events in the present study were measured in the same categories as adverse events but without a temporal relationship with medication use; nonetheless, the presence of clinical events signals the likelihood of poor outcomes. Clinical events, including falls/fractures and extrapyramidal reactions, have been associated with more rapid cognitive and functional decline, earlier institutionalization, and increased mortality. ${ }^{28-31}$ Yearly costs attributed to falls in the United States are estimated at $\$ 668.5$ million, ${ }^{32}$ and fall accidents are the leading cause of Alzheimer disease-related hospitalization, accounting for $26 \%$ of U.S. hospitalizations. ${ }^{33}$

In addition to falls/fractures, use of antipsychotic medications in elderly dementia patients is associated with increased risk of severe side effects, including cerebrovascular events, extrapyramidal reactions, cardiovascular disease, and mortality. ${ }^{19-24}$ In a cost-benefit analysis of antipsychotic treatment for dementia, patients treated with an atypical antipsychotic had significantly higher health care costs, but no benefit in quality-adjusted life-years or functional measures compared with placebo. ${ }^{34}$ Medications commonly used off-label to treat DRP show no cost-offset benefits $^{34}$ and are associated with adverse effects that lead to hospitalization, lower cognitive functioning, and institutionalization. ${ }^{19-24}$ When nonpharmacologic treatments for psychosis fail, patients and providers are left with limited care options. Safe, effective treatments for DRP are lacking in the current treatment landscape.

Results from the present study confirm the cost of dementia found in other studies and highlight how the occurrence of psychosis among dementia patients leads to additional HCRU and costs. Total all-cause health care costs in the post-index period were estimated at $\$ 12,026$ among patients with dementia only. This was similar to the annual direct health care costs of $\$ 14,508$ estimated for patients with Alzheimer disease and other dementias. ${ }^{35}$ In the present study, patients with DRP had significantly higher all-cause HCRU and costs compared with dementiaonly patients. Total mean all-cause costs in the post-index period were 1.8 times higher among DRP than dementiaonly patients (\$21,657 vs. \$12,026). Inpatient costs were the major cost driver and were 2.8 times higher among DRP versus dementia patients without psychosis. Emergency 


\section{FIGURE 4 Mean Health Care Costs During the 12-Month Pre-Index and Post-Index Periods ${ }^{\mathrm{a}}$}

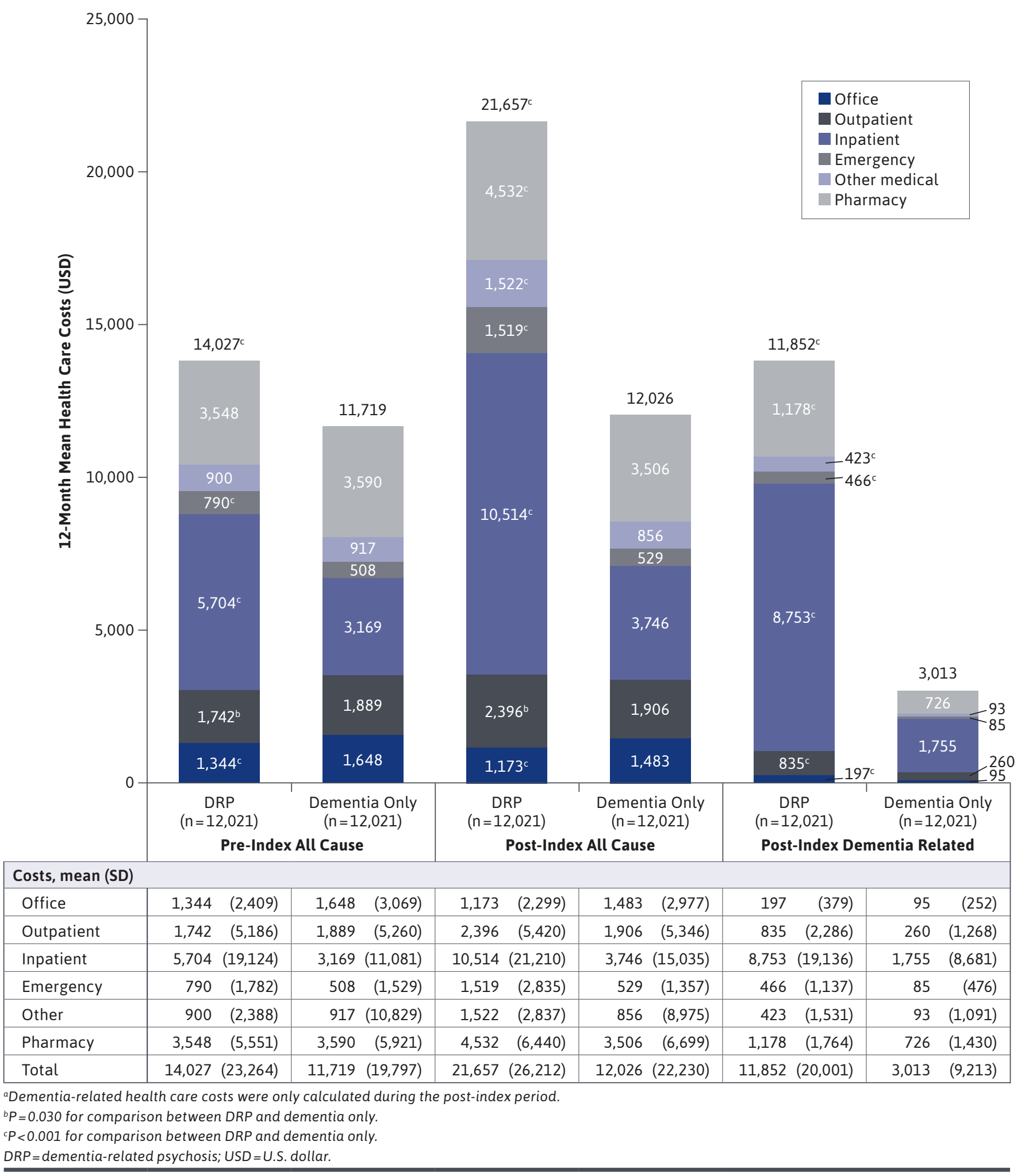


costs were 2.9 times higher among DRP compared with dementia-only patients.

Additionally, patients with DRP had greater dementiarelated HCRU and costs compared with dementia-only patients. Dementia-related costs, on average, accounted for $54.7 \%$ of all-cause post-index costs in patients with DRP and $25.1 \%$ of all-cause costs among patients with dementia only. Studies that address the costs associated with DRP are lacking; however, 2 studies found that direct health care costs increased with worsening neuropsychiatric symptoms in patients with dementia, measured using a 96-point scale. ${ }^{36,37}$ In Murman et al. (2005), a 1-point worsening of the neuropsychiatric inventory score was associated with an incremental direct cost increase between \$247 and \$409 annually in 2001 U.S. dollars. ${ }^{36}$ Similarly, in a study of dementia patients by Herrmann et al. (2006), each 1-point increase in the neuropsychiatric inventory score was associated with a \$30 per month increase in direct health care costs. ${ }^{38}$ Even incremental improvements in psychosis should result in health care savings.

This study documented the significant clinical and economic burden associated with DRP. The high costs of dementia-related spending as a proportion of all-cause health care spending signifies the significant economic burden placed on health care payers and patients with dementia, particularly those with DRP, and highlights the need for more effective treatments and interventions that can delay or lessen the severity of DRP. Future research is needed to delineate patient factors associated with increased HCRU and costs to provide insight for targeting effective treatment strategies as they become available.

\section{LIMITATIONS}

Certain limitations are common in claims database analyses, and results described should be interpreted with consideration of such limitations. The presence of a diagnosis code on a medical claim is not proof positive of disease as it could be incorrectly coded or included as rule-out criteria rather than actual disease. In addition, behavioral health diagnoses might not be consistently coded on claims due to stigma or concerns about the effects of a major psychological issue documented in the patient's medical record. To mitigate these concerns, more than 1 diagnosis or a combination of $\geq 1$ diagnosis and $\geq 1$ pharmacy fill for a therapeutic medication was required to identify patients with DRP and dementia. Additionally, patients with behavioral health diagnoses such as bipolar disorder were excluded from the sample; however, these diseases are underdiagnosed, and it is possible that patients with these conditions were inadvertently included in the study sample.
Clinical events were not measured with any temporal relationship to antipsychotic consumption and, thus, should not be interpreted as resulting from antipsychotic therapy. Additionally, clinical events are likely underreported. For example, a fall may not have been serious enough to require a provider visit and, therefore, would not appear on a medical claim.

This study also required $\geq 12$ months of continuous health plan enrollment before and after evidence of diagnosis/treatment, which may have introduced selection bias. Patients who disenrolled (including those who died) before meeting enrollment requirements were excluded, potentially resulting in healthier patients retained for the study.

Additionally, this study did not provide the complete economic journey of the DRP patient, and costs associated with admission to long-term or chronic care were not included, therefore costs may be underestimated.

Finally, this study was conducted in a large U.S. managed care population, and results may not be generalizable to other populations, such as Medicaid for fee-for-service Medicare beneficiaries or all patients with a DRP or dementia diagnosis.

\section{Conclusions}

This study identified and recognized the high rates of clinical events, HCRU, and associated health care costs among dementia patients newly diagnosed with psychosis. Compared with patients with dementia only, those with DRP had a higher comorbidity score and were more likely to suffer from depressive, anxiety, and insomnia disorders. Patients with DRP had significantly more clinical events than patients with dementia only, most notably falls/fractures and sedation. DRP patients had consistently higher all-cause and dementia-related HCRU and costs compared with patients with dementia only. Higher HCRU and costs were driven by acute care received in emergency and inpatient settings. These results reflect the unmet need and urgency for new treatment options that may reduce the clinical and economic burden in this population, which would further help patients, caregivers, health care professionals, and payers reduce the costs and stress associated with DRP. With a growing population of elderly patients with DRP whose current treatment choices may be associated with higher rates of clinical events and increased HCRU and costs, the development of effective treatment options is an important opportunity that could have a direct effect upon those who pay for and provide DRP care. 


\section{DISCLOSURES}

This study was funded by Acadia Pharmaceuticals, which participated in the study design, interpretation of study results, and critical review of the manuscript. Abler, Skoog, and Rashid were employees of Acadia Pharmaceuticals at the time this study was conducted. Frazer and Halpern were employees of Optum at the time this study was conducted and were funded by Acadia Pharmaceuticals to conduct the study.

\section{ACKNOWLEDGMENTS}

Deja Scott-Shemon, MPH, an employee of Optum, provided medical writing assistance. Sharanya Murali and Megan Sipper, employees of Optum, provided project management assistance.

\section{REFERENCES}

1. Boustani M, Peterson B, Hanson L, Harris R, Lohr KN; U.S. Preventative Services Task Force. Screening for dementia in primary care: a summary of the evidence for the U.S. Preventive Services Task Force. Ann Intern Med. 2003;138(11):927-37.

2. Bradford A, Kunik ME, Schulz P, Williams SP, Singh H. Missed and delayed diagnosis of dementia in primary care: prevalence and contributing factors. Alzheimer Dis Assoc Disord. 2009;23(4):306-14.

3. Kotagal V, Langa KM, Plassman BL, et al. Factors associated with cognitive evaluations in the United States. Neurology. 2015;84(1):64-71.

4. Taylor DH, Jr., Ostbye T, Langa KM, Weir D, Plassman BL. The accuracy of Medicare claims as an epidemiological tool: the case of dementia revisited. J Alzheimers Dis. 2009;17(4):807-15.

5. Amjad H, Roth DL, Sheehan OC, Lyketsos CG, Wolff JL, Samus QM. Underdiagnosis of dementia: an observational study of patterns in diagnosis and awareness in U.S. older adults. J Gen Intern Med. 2018;33(7):1131-38.
6. Matthews KA, Xu W, Gaglioti AH, et al. Racial and ethnic estimates of Alzheimer's disease and related dementias in the United States (2015-2060) in adults aged $\geq 65$ years. Alzheimers Dement. 2019;15:17-24.

7. Hurd MD, Martorell P, Delavande A, Mullen KJ, Langa KM. Monetary costs of dementia in the United States. N Engl J Med. 2013;368(14):1326-34.

8. Alzheimer's Association. Alzheimer's disease and chronic health conditions: the real challenge for 21st century Medicare. Accessed February 10, 2021. www.alz.org/national/documents/ report chroniccare.pdf

9. Cummings J, Ballard C, Tariot P, et al. Pimavanserin: potential treatment for dementia-related psychosis. J Prev Alzheimers Dis. 2018;5(4):253-58.

10. Ballard CG, Gauthier S, Cummings JL, et al. Management of agitation and aggression associated with Alzheimer disease. Nat Rev Neurol. 2009;5(5):245-55.

11. Peters ME, Schwartz S, Han D, et al. Neuropsychiatric symptoms as predictors of progression to severe Alzheimer's dementia and death: the Cache County Dementia Progression Study. Am J Psychiatry. 2015;172(5):460-65.

12. Habiger TF, Achterberg WP, Flo E, Husebo BS. Psychosis symptoms in nursing home residents with and without dementia: cross-sectional analyses from the COSMOS study. Int J Geriatr Psychiatry. 2019;34(5):683-91.

13. Stern Y, Tang MX, Albert MS, et al. Predicting time to nursing home care and death in individuals with Alzheimer disease. JAMA. 1997;277(10):806-12.

14. Gonzalez-Salvador T, Lyketsos CG, Baker A, et al. Quality of life in dementia patients in long-term care. Int J Geriatr Psychiatry. 2000;15(2):181-89.

15. Gonzalez-Salvador MT, Arango C, Lyketsos CG, Barba AC. The stress and psychological morbidity of the Alzheimer patient caregiver. Int J Geriatr Psychiatry. 1999;14(9):701-10.
16. Steele C, Rovner B, Chase GA, Folstein M. Psychiatric symptoms and nursing home placement of patients with Alzheimer's disease. Am J Psychiatry. 1990;147(8):1049-51.

17. Maust DT, Kales HC, McCammon RJ, Blow FC, Leggett A, Langa KM. Distress associated with dementia-related psychosis and agitation in relation to healthcare utilization and costs. Am J Geriatr Psychiatry. 2017;25(10):1074-82.

18. Gerlach LB, Kales HC. Managing behavioral and psychological symptoms of dementia. Psychiatr Clin North Am. 2018;41(1):127-39.

19. Reus VI, Fochtmann LJ, Eyler AE, et al. The American Psychiatric Association Practice Guideline on the Use of Antipsychotics to Treat Agitation or Psychosis in Patients With Dementia. Am J Psychiatry. 2016;173(1):543-46.

20. Aarsland D, Sharp S, Ballard C. Psychiatric and behavioral symptoms in Alzheimer's disease and other dementias: etiology and management. Curr Neurol Neurosci Rep. 2005;5(5):345-54.

21. Pijnenburg YA, Sampson EL, Harvey RJ, Fox NC, Rossor MN. Vulnerability to neuroleptic side effects in frontotemporal lobar degeneration. Int J Geriatr Psychiatry. 2003;18(1):67-72.

22. Stinton C, McKeith I, Taylor JP, et al. Pharmacological management of Lewy body dementia: a systematic review and meta-analysis. Am J Psychiatry. 2015;172(8):731-42.

23. Mittal V, Kurup L, Williamson D, Muralee S, Tampi RR. Risk of cerebrovascular adverse events and death in elderly patients with dementia when treated with antipsychotic medications: a literature review of evidence. Am J Alzheimers Dis Other Demen. 2011;26(1):10-28.

24. Steinberg M, Lyketsos CG. Atypical antipsychotic use in patients with dementia: managing safety concerns. Am J Psychiatry. 2012;169(9):900-06. 
25. Quan H, Li B, Couris CM, et al. Updating and validating the Charlson comorbidity index and score for risk adjustment in hospital discharge abstracts using data from 6 countries. Am J Epidemiol. 2011;173(6):676-82.

26. Bayliss EA, Ellis JL, Shoup JA, Zeng C, McQuillan DB, Steiner JF. Association of patient-centered outcomes with patientreported and ICD-9-based morbidity measures. Ann Fam Med. 2012;10(2):126-33.

27. U.S. Bureau of Labor Statistics. CPI for all urban consumers (CPI-U). Series ID: CUUR0000SAM. Accessed February 10, 2021. http://data.bls.gov/cgi-bin/ surveymost?cu

28. Stern Y Albert M, Brandt J, et al. Utility of extrapyramidal signs and psychosis as predictors of cognitive and functional decline, nursing home admission, and death in Alzheimer's disease. Neurology. 1994;44(12):2300.

29. Myers AH, Baker SP, Van Natta ML, Abbey H, Robinson EG. Risk factors associated with falls and injuries among elderly institutionalized persons. Am J Epidemiol. 1991;133(11):1179-90.
30. Portet F, Scarmeas N, Cosentino S, Helzner EP, Stern Y. Extrapyramidal signs before and after the diagnosis of incident Alzheimer disease in a prospective population study. Arch Neurol. 2009;66(9):1120-26.

31. Haan MN, Jagust WJ, Galasko D, Kaye J. Effect of extrapyramidal signs and Lewy bodies on survival in patients with Alzheimer disease. Arch Neurol. 2002;59(4):588-93.

32. Burns ER, Stevens JA, Lee R. The direct costs of fatal and non-fatal falls among older adults - United States. J Safety Res. 2016;58:99-103.

33. Alzheimer's Association. 2016 Alzheimer's disease facts and figures. Alzheimers Dement. 2016;12:459-509.

34. Rosenheck RA, Leslie DL, Sindelar JL, et al. Cost-benefit analysis of secondgeneration antipsychotics and placebo in a randomized trial of the treatment of psychosis and aggression in Alzheimer disease. Arch Gen Psychiatry. 2007;64(11):1259-68.
35. Deb A, Sambamoorthi U, Thornton JD, Schreurs B, Innes K. Direct medical expenditures associated with Alzheimer's and related dementias (ADRD) in a nationally representative sample of older adults - an excess cost approach. Aging Ment Health. 2018;22(5):619-24.

36. Murman DL, Colenda CC. The economic impact of neuropsychiatric symptoms in Alzheimer's disease: can drugs ease the burden? Pharmacoeconomics. 2005;23(3):227-42.

37. Hermanowicz N, Edwards K. Parkinson's disease psychosis: symptoms, management, and economic burden. Am J Manag Care. 2015;21(10 Suppl):s199-206.

38. Herrmann N, Lanctot KL, Sambrook $\mathrm{R}$, et al. The contribution of neuropsychiatric symptoms to the cost of dementia care. Int J Geriatr Psychiatry. 2006;21(10):972-76. 